\title{
A NOTE ON TANGENT BUNDLES
}

\author{
KENICHI SHIRAIWA
}

Dedicated to Professor K. Noshiro for his 60 th birthday

The tangent bundle of a differentiable manifold is an important invariant of a differentiable structure. It is determined neither by the topological structure nor by the homotopy type of a manifold. But in some cases tangent bundles depend only on the homotopy types of manifolds.

In this note we shall show that homotopy spheres and homotopy real projective spaces have homotopically equivalent tangent bundles respectively. Also, the action of $\Theta_{n}$, the group of the homotopy spheres, on an oriented smooth manifold by the connected sum does not have an effect on the structure of the tangent bundle $(n \geqq 5)$.

1. Let $M^{n}$ be a differentiable manifold of dimension $n$. Let $\xi$, $\xi^{\prime}$ be vector bundles over $M^{n}$. If $\xi$ is equivalent (or isomorphic) to $\xi^{\prime}$, then we shall denote it by $\xi \approx \xi^{\prime}$. Let $\tau\left(M^{n}\right)$ be the tangent bundle of $M^{n}$.

THEOREM 1. Let $\Sigma^{n}$ be a homotopy $n$-sphere. Let $f: S^{n} \rightarrow \Sigma^{n}$ be an orientation preserving homotopy equivalence of the standard $n$-sphere $S^{n}$ onto $\Sigma^{n}$. Then $f^{*}\left(\tau\left(\Sigma^{n}\right)\right) \approx \tau\left(S^{n}\right)$. In other words, $f$ is covered by a bundle map $\tilde{f}$ of $\tau\left(S^{n}\right)$ onto $\tau\left(\sum^{n}\right)$.

Remark. If $n$ is even and $n \neq 2(\bmod 8)$, then this is a consequence of a theorem of Takeuchi [11].

Proof. If $n \leqq 7$, Theorem 1 is well known and derived by the similar argument that follows. Therefore, we assume $n \geqq 8$. Let $\tau^{\prime}=f^{*} \tau\left(\Sigma^{n}\right)$ and $\tau=\tau\left(S^{n}\right)$. We shall show that $\tau \approx \tau^{\prime}$. Let $\xi$ be an oriented $n$-plane bundle over $S^{n}$. Let $\alpha(\xi) \in \pi_{n-1}(S O(n))$ be the characteristic class of $\xi$. By the classification theorem (Steenrod [10]) it is sufficient to prove that $\alpha(\tau)=\alpha\left(\tau^{\prime}\right)$. Let $i: S O(n) \rightarrow S O(n+1)$ be the inclusion, and let $i_{*}: \pi_{n-1}(S O(n)) \rightarrow \pi_{n-1}(S O(n+1))$ be the induced

Received June 20, 1966. 
homomorphism. Then $\alpha\left(\tau^{\prime}\right) \in \operatorname{Ker} i_{*}$, since homotopy spheres are stably parallelizable (Kervaire-Milnor [6]). By a result of Kervaire [7], (See also Steenrod [10]), $\operatorname{Ker} i_{*}=Z$, the group of the integers, if $n$ is even, and $\operatorname{Ker} i_{*}=Z_{2}$, the group of integers mod 2, if $n$ is odd. And in both cases $\alpha(\tau)$ generates Ker $i_{*}$. It is also easy to see that $\operatorname{Ker} i_{*}$ is a direct summand of $\pi_{n-1}(S O(n))$.

Case 1. Let $n$ be odd. Then $\operatorname{Ker} i_{*}=Z_{2}$, and $\alpha(\tau) \neq 0$. Therefore, it is sufficient to prove that $\alpha\left(\tau^{\prime}\right) \neq 0$, that is, $\Sigma^{n}$ is not parallelizable for $n \geqq 8$.

Lemma 1 (A. Dold). If $\Sigma^{n}$ is paratlelizable, then $S^{n}$ is an $H$-space.

Since we do not find the proof of Dold, we shall give a proof of the lemma. (Cf. Adams [1])

Proof of Lemma 1. Let $\Delta \subset \Sigma^{n} \times \Sigma^{n}$ be the diagonal. Let $p: \Sigma^{n} \times \Sigma^{n} \rightarrow \Sigma^{n}$ be the projection on the first factor. Then it is well known that there exists a closed tubular neighborhood $U$ of $\Delta$ such that $p_{1}=p \mid U: U \rightarrow \Sigma^{n}$ is equivalent to a suitable closed disk bundle associated to the tangent bundle $\tau\left(\Sigma^{n}\right)$. Since $\Sigma^{n}$ is parallelisable, $\tau\left(\Sigma^{n}\right)$ is trivial. Therefore, there exists a homeomorphism $h: U \approx \Sigma^{n} \times D^{n}$ such that

a)

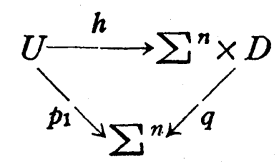

is commutative, where $D^{n}$ is a closed $n$-disk and $q$ is the projection.

b) $h$ maps $\Delta$ onto $\Sigma^{n} \times\{0\}$ in such a way that $h(x, x)=(x, 0)$ for $x \in \Sigma^{n}$, where $0 \in D^{n}$ is the origin of the disk $D^{n}$.

Let $\varphi: D^{n} \rightarrow S^{n}$ be a map such that

(i) $\varphi\left(\partial D^{n}\right)=x_{1} \in S^{n}$, where $\partial D^{n}=S^{n-1}$ is the boundary of the disk $D^{n}$,

(ii) $\varphi(0)=x_{0}=$ the base point of $S^{n}$, and

(iii) $\varphi$ is of degree 1 .

Let $k: \Sigma^{n} \times \Sigma^{n} \rightarrow S^{n}$ be a Pontrjagin-Thom map defined by

$$
\begin{aligned}
& k\left(\Sigma^{n} \times \Sigma^{n}-U\right)=x_{1}, \\
& k \mid U=\varphi \cdot r \cdot h \text {, where } r: \Sigma^{n} \times D^{n} \rightarrow D^{n} \text { is the projection. }
\end{aligned}
$$

Then it is easy to see that $k \mid\{y\} \times \sum^{n}:\{y\} \times \Sigma^{n} \rightarrow S^{n}$ is of degree 1 for each $y \in \Sigma^{n}$ and $k(\Delta)=x_{0}$. 
Now $\pi_{n}\left(\sum^{n} \times \sum^{n}\right)=\pi_{n}\left(\Sigma^{n}\right)+\pi_{n}\left(\sum^{n}\right)=Z+Z$ is generated by $i_{1}: S^{n} \rightarrow$ $\Sigma^{n} \times y_{0} \subset \Sigma^{n} \times \Sigma^{n}$ and $i_{2}: S^{n} \rightarrow y_{0} \times \Sigma^{n} \subset \Sigma^{n} \times \Sigma^{n}$, where $i_{1}$ and $i_{2}$ are maps of degree 1. Let $i: S^{n} \rightarrow \Delta \subset \Sigma^{n} \times \Sigma^{n}$ be a map of degree 1. Then $\{i\}=\left\{i_{1}\right\}+\left\{i_{2}\right\}$, where the bracket means a homotopy class.

Let $k_{*}: \pi_{n}\left(\Sigma^{n} \times \Sigma^{n}\right) \rightarrow \pi_{n}\left(S^{n}\right)$ be the induced homomorphism of $k$. Then $k_{*}\left(\left\{i_{2}\right\}\right)=\iota_{n}$, the generator of $\pi_{n}\left(S^{n}\right)=Z$, and $k_{*}\left(\left\{i_{1}\right\}+\left\{i_{2}\right\}\right)=k_{*}(\{i\})=0$. Therefore, $k_{*}\left(\left\{i_{2}\right\}\right)=-\imath_{n}$. Thus $k: \Sigma^{n} \times \Sigma^{n} \rightarrow S^{n}$ is of type $(-1,1)$.

Let $j, j^{\prime}: S^{n} \rightarrow \Sigma^{n}$ be maps of degree $-1,1$ respectively, which preserve base points. Let $m: S^{n} \times S^{n} \rightarrow S^{n}$ be defined by $m=k \circ\left(j \times j^{\prime}\right)$. Then $m$ is of type $(1,1)$ and preserves base points. Thus $m$ defines an $H$-space structure on $S^{n}$.

Corollary. (Adams [1]) Homotopy n-spheres are parallelizable if and only if $n=1,3$, or 7 .

Proof. By the result of Adams [1], $S^{n}$ is an $H$-space if and only if $n=1$, 3 , or 7 . Hence, if $\Sigma^{n}$ is parallelizable, $n=1,3$, or 7 . Conversely, a homotopy $n$-sphere is parallelisable for $n=1,3,7$, since $\pi_{n-1}(S O(n))=0$ in this case.

Now the proof of Theorem 1. Case 1 is complete.

Case 2. Let $n$ be even. Then $\operatorname{Ker} i_{*}=Z$ and $\alpha(\tau)$ generates $\operatorname{Ker} i_{*}$. Since $\alpha\left(\tau^{\prime}\right) \in \operatorname{Ker} i_{*}, \alpha\left(\tau^{\prime}\right)=m \alpha(\tau)$ for some integer $m$. We shall show $m=1$.

Let $p: S O(n) \rightarrow S O(n) / S O(n-1)=S^{n-1}$ be the projection. Then it is well known that $p_{*}: \pi_{n-1}(S O(n)) \rightarrow \pi_{n-1}\left(S^{n-1}\right)=Z$ maps Ker $i_{*}$ monomorphically onto $2 \pi_{n-1}\left(S^{n-1}\right) \subset \pi_{n-1}\left(S^{n-1}\right)$. (Steenrod [10]) Now we need the following lemma.

Lemмa 2. Let $\xi$ be an oriented n-plane bundle over $S^{n}$. Then the Euler class $X(\xi)=-p_{*}(\alpha(\xi)) \in H^{n}\left(S^{n}, \pi_{n-1}\left(S^{n-1}\right)\right)=\pi_{n-1}\left(S^{n-1}\right)$.

Proof. Let $\pi: E \rightarrow S^{n}, \pi^{\prime}: E^{\prime} \rightarrow S^{n}$ be the associated principal bundle and the $(n-1)$-sphere bundle of $\xi$ respectively. Let $q: E \rightarrow E^{\prime}$ be the associated principal map. Then



is commutative and the restriction of $q$ on the fibre is equivalent to $\left.p: S O^{\prime} n\right) \rightarrow S^{n-1}$.

Now consider the following commutative diagram; 


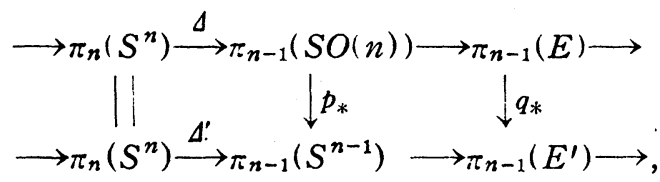

where each row is the exact sequence of the homotopy groups of the bundles $E$ and $E^{\prime}$ respectively. Then $\Delta\left(\iota_{n}\right)=\alpha(\xi)$, and $-p_{*}(\alpha(\xi))=-p_{*} \Delta\left(\iota_{n}\right)=-\Delta^{\prime}\left(\iota_{n}\right)$. But $-\Delta^{\prime}\left(\iota_{n}\right)$ is identified with the Euler class $X(\xi)$ of $\xi$. (Cf. Steenrod [10]). This completes the proof of the lemma.

Returning to the proof of Theorem 1, we get $X\left(\tau^{\prime}\right)=m X(\tau)$ by our assumption $\alpha\left(\tau^{\prime}\right)=m \alpha(\tau)$ and Lemma 2. But the Euler class of the tangent bundle of a differentiable manifold is identified with the Euler characteristic of the manifold. Thus $X(\tau)=X\left(\tau^{\prime}\right)=$ twice of the generator of $H^{n}\left(S^{n}, \pi_{n-1}\left(S^{n-1}\right)\right)$, and this implies $m=1$.

2. Let $M^{n}$ be a connected closed oriented differentiable manifold of dimension $n$. Let $\Sigma^{n}$ be a homotopy $n$-sphere.

Theorem 2. Let $n \geqq 5$. Let $M^{n} \# \Sigma^{n}$ be the connected sum of $M^{n}$ and $\Sigma^{n}$. Then there exists an orientation preserving homeomorphism $f: M^{n} \rightarrow M^{n} \# \sum^{n}$ such that $f^{*}\left(\tau\left(M^{n} \# \Sigma^{n}\right)\right) \approx \tau\left(M^{n}\right)$.

Before proving Theorem 2, we need the following two lemmas.

Lemma 3. Let $M_{1}, M_{2}$ be closed oriented smooth n-manifolds. Let $f: M_{1} \rightarrow M_{2}$ be a map satisfying the following conditions.

(i) There exists a neighborhood $U(p)$ of $p \in M_{1}$ such that $f \mid U(p): U(p) \rightarrow M_{2}$ is a differentiable imbedding.

(ii) $f$ is covered by a bundle map $\tau: \tau\left(M_{1}\right) \rightarrow \tau\left(M_{2}\right)$.

Then there exists a bundle map $F: \tau\left(M_{1}\right) \rightarrow \tau\left(M_{2}\right)$ covering $f$ such that $(a)$ $F=d f$, the differential of $f$ on $\tau\left(M_{1}\right) \mid V(p)$, where $V(p)$ is a neighborhood of $p$ contained in $U(p)$, and $(b) F=\tilde{f}$ on $\tau\left(M_{1}\right) \mid\left(M_{1}-U(p)\right)$.

Proof. Let $\bar{U}_{1} \subset U(p)$ be a closed neighborhood of $p$ diffeomorphic to the closed disk $D^{n}$ of radius 1 . Let $V(p) \subset \bar{U}_{1}$ be the neighborhood corresponding to the open disk of radius $1 / 2$. Since $\bar{U}_{1}$ is contractible, $\tau\left(M_{1}\right) \mid \bar{U}_{1}$ is trivial and $\tau\left(M_{1}\right) \mid \bar{U}_{1}=\bar{U}_{1} \times R^{n}$, where $R^{n}$ is the real $n$-space. Since $f \mid U(p)$ is a differentiable imbedding, $\tau\left(M_{2}\right) \mid f\left(\bar{U}_{1}\right)=f\left(\bar{U}_{1}\right) \times R^{n}$.

Using the above identification, we can represent $\widetilde{f}$ as follows; 


$$
\widetilde{f}: \bar{U}_{1} \times R^{n} \rightarrow f\left(\bar{U}_{1}\right) \times R^{n}
$$

is given by $\widetilde{f}(x, y)=(f(x), g(x) y)$, where $g: \ddot{U}_{1} \rightarrow G L(n, R)$ is a suitable map to the full linear group of $R^{n}$, and thus $g(x)$ operates on $R^{n}$. It is clear that $g \mid \partial \bar{U}_{1}$, where $\partial \bar{U}_{1}$ is the boundary of $\bar{U}_{1}$, is homotopic to zero. Since df is a bundle map on $\tau\left(M_{1}\right) \mid \bar{U}_{1}$ covering $f, d f(x, y)=\left(f(x), g^{\prime}(x) y\right)$ for some $g^{\prime}: \bar{U}_{1} \rightarrow G L(n, R)$. (Essentially $g^{\prime}(x)$ is $\left.d f_{x}\right) g^{\prime} \mid \partial V(p)$ is homotopic to zero as before. Hence $g \mid \partial \bar{U}_{1}$ is homotopic to $g^{\prime} \mid \partial V(p)$. Using a homotopy between $g$ and $g^{\prime}$, we can construct a map $g^{\prime \prime}: \bar{U}_{1} \rightarrow G L(n, R)$ such that

(i) $g^{\prime \prime} \mid \partial \bar{U}_{1}=g$, and

(ii) $g^{\prime \prime} \mid V(p)=g^{\prime}$.

Define $F: \tau\left(M_{1}\right) \rightarrow \tau\left(M_{2}\right)$ by

(a) $F=\widetilde{f}$ on $\tau\left(M_{1}\right) \mid\left(M_{1}-\bar{U}_{1}\right)$, and

(b) $F(x, y)=\left(f(x), g^{\prime \prime}(x) y\right)$ on $\tau\left(M_{1}\right) \mid \bar{U}_{1}$.

Then $F$ satisfies the required properties of the lemma.

Lemma 4. Let $M_{1}, M_{2}, N$ be closed connected oriented smooth manifolds of dimension n. Let $f: M_{1} \rightarrow M_{2}$ be an orientation preserving homeomorphism satisfy. ing the following conditions:

(i) $f$ is a diffeomorphism on a neighborhood $U(p)$ of $p \in M_{1}$.

(ii) $f^{*}\left(\tau\left(M_{2}\right)\right) \approx \tau\left(M_{1}\right)$.

Then there exists an orientation preserving homeomorphism $h: M_{1} N^{*} N \rightarrow M_{2} \# N$ such that $h$ is covered by a bundle map $\left.\tau M_{1} \# N\right) \rightarrow \tau\left(M_{2} \# N\right)$, i.e. $h^{*}\left(\tau\left(M_{2} \# N\right)\right) \approx \tau\left(M_{1} \# N\right)$.

Proof. First observe that $f$ satisfies the conditions of Lemma 3.

Let $D^{n}$ be the unit disk. Let $k ; D^{n} \rightarrow M_{1}$ be an orientation preserving smooth imbedding such that $k\left(D^{n}\right) \subset V(p)$, where $V(p)$ is the neighborhood given in Lemm 3. Then $f \circ k: D^{n} \rightarrow M_{2}$ is an orientation preserving smooth imbedding.

Let $r: D^{n} \rightarrow N$ be a smooth imbedding which reverses orientation. Then $M_{1} \# N$ is obtained from the disjoint union $\left(M_{1}-k(0)\right)+(N-r(0))$ by identifying $k(t x)$ with $r((1-t) x)$ for each $x \in S^{n-1}=\partial D^{n}$ and each $0<t<1$. In other words, $y \in k\left(\right.$ Int $\left.D^{n}-0\right)$ is identified with $r \circ \phi \circ k(y)$, where $\phi:$ Int $D^{n}-0 \rightarrow \operatorname{Int} D^{n}-0$ is a diffeomorphism defined by $\phi(t x)=(1-t) x$ for $x \in S^{n-1}$ and $0<t<1$, and Int $D^{n}$ is the interior of $D^{n}$.

Now $\tau\left(M_{1} \# N\right)$ is obtained from the disjoint union $\left(\tau\left(M_{1}\right)\right.$ - the fibre over $k(0))+(N$ - the fibre over riol) by identifying $(k(t x), y)$ with $(r((1-t) x)$, 
$\left.d r \circ d \phi \circ d k^{-1}(y)\right)$, where $y$ is a tangent vector at $k(t x)$, for each $x \in S^{n-1}$ and $0<t<1$.

Similary, $M_{2} \# N$ is obtained from the disjoint union $\left(M_{2}-f \circ k(0)+\left(N-r^{\prime}(0)\right)\right.$ by identifying $f \circ k(t x)$ with $r((1-t) x)$ for each $x \in S^{n-1}$ and $0<t<1$. And $\tau\left(M_{2} \# N\right)$ is obtained from the disjoint union $\left(\tau\left(M_{2}\right)\right.$ - the fibre over $\left.f \circ k(0)\right)+$ $(N-$ the fibre over $r(0))$ by identifying $\left(f \circ k(t x), y^{\prime}\right)$ with $(r((1-t) x)$, $\left.d r \circ d \phi^{\circ} d(f \circ k)^{-1}\left(y^{\prime}\right)\right)$, where $y^{\prime}$ is a tangent vector at $f \circ k(t x)$.

Define $h: M_{1} \# N \rightarrow M_{2} \# N$ by

$$
\begin{aligned}
& h \mid\left(M_{1}-k(0)\right)=f, \text { and } \\
& h \mid(N-r(0))=\text { the identity map. }
\end{aligned}
$$

Then $h$ is an orientation preserving homeomorphism.

Let $F: \tau\left(M_{1}\right) \rightarrow \tau\left(M_{2}\right)$ be the bundle map covering $f$ given by Lemma 3 such that $F=d f$ on $\tau\left(M_{1}\right) \mid V(p)$. Define $H: \tau\left(M_{1} \# N\right) \rightarrow \tau\left(M_{2} \# N\right)$ by

$$
\begin{aligned}
& \left.H\right|_{\tau}\left(M_{1}\right) \text { - the fibre over } k(0)=F \text {, and } \\
& \left.H\right|_{\tau}(N) \text { - the fibre over } r(0)=\text { the identity map. }
\end{aligned}
$$

Then it is easy to see that $H$ is a bundle map covering $h$.

Proof of Theorem 2. Since $M^{n}$ is diffeomorphic to $M^{n} \# S^{n}$, we shall prove the existence of an orientation preserving homeomorphism $h: M^{n} \# S^{n} \rightarrow M^{n} \# \Sigma^{n}$ which is covered by a bundle map $\tau\left(M_{1} \# S^{n}\right) \rightarrow \tau\left(M_{2} \# \Sigma^{n}\right)$. It is well known that there exists an orientation preserving homeomorphism $f: S^{n} \rightarrow \Sigma^{n}$ which is a diffeomorphism except one point for $n \geqq 5$.

Theorem 1 implies $f^{*}\left(\tau\left(\sum^{n}\right)\right) \approx \tau\left(S^{n}\right)$. Thus the conditions of Lemma 4 is satisfied, and Theorem 2 follows.

COROLlary 1. Let $M_{1}, M_{2}$ be connected closed oriented smooth n-manifolds whose underlying topological manifolds are homeomorphic $(n \geqq 5)$. Suppose for any orientation preserving homeomorphism $f: M_{1} \rightarrow M_{2}, f^{*}{ }_{\tau}\left(M_{2}\right)$ is not equivalent to $\tau\left(M_{1}\right)$. Then $M_{1}$ is not diffeomorphic to the connected sum of $M_{2}$ and $\Sigma^{n}$ for any homotopy sphere $\Sigma^{n}$.

COROLLARY 2. If $n \geqq 5$, then $\left(M^{n} \# \Sigma^{n}\right) \times R^{k}$ is diffeomorphic to $M^{n} \times R^{k}$ for $k \geqq n+2$ and for any homotopy sphere $\Sigma^{n}$.

This is clear by Mazur [8]. 
3. Let $M^{n}$ be a closed differentiable $n$-manifold which is homotopically equivalent to the real projective $n$-space $P^{n}$. Then we shall call $M^{n}$ a homotopy real projective $n$-space (Cf. [4], [9]).

Theorem 3. Let $M^{n}$ be a homotopy real projective $n$-space. Let $f: P^{n} \rightarrow M^{n}$ be a homotopy equivalence. Then $f^{*}\left(\tau\left(M^{n}\right)\right)$ is equivalent to $\tau\left(P^{n}\right)$.

This is a generalization of Lemma 4 of Hirsch-Milnor [4].

Proof. By. Theorem (3.6) of Atiyah [3], $J\left(\tau\left(P^{n}\right)\right)=J\left(f^{*}(\tau(M))\right)$. By (6.3) of Adams [2], $J: K O\left(P^{n}\right) \rightarrow J\left(P^{n}\right)$ is an isomorphism: Thus $f^{*}\left(\tau\left(M^{n}\right)\right)$ is stably equivalent to $\tau\left(P^{n}\right)$. If $n$ is odd, Corollary (1.11) of James-Thomas [5] implies that $f^{*}\left(\tau\left(M^{n}\right)\right) \approx \tau\left(P^{n}\right)$. Therefore, it remains the case $n$ is even.

Let $n$ be even. Let $\Sigma^{n}$ be the universal covering manifold of $M^{n}$. Then $\Sigma^{n}$ is a homotopy sphere and $f$ is covered by a homotopy equivalence $\tilde{f}: S^{n} \rightarrow \Sigma^{n}$. Thus

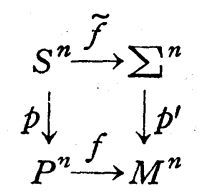

is commutative, where $p, p^{\prime}$ are projections. Therefore, $p^{\prime *}\left(\tau\left(M^{n}\right)\right) \approx \tau\left(\sum^{n}\right)$ and $p^{*}\left(\tau\left(P^{n}\right)\right) \approx \tau\left(S^{n}\right)$. Since $\tilde{f}^{*}\left(\tau\left(\sum^{n}\right)\right) \approx \tau\left(S^{n}\right)$ by Theorem $1, \tilde{f}^{*} p^{\prime *}\left(\tau\left(M^{n}\right)\right)$ $\approx p^{*}\left(\tau\left(P^{n}\right)\right) \approx p^{*} f^{*}\left(\tau\left(M^{n}\right)\right)$.

Now our proof proceeds as the one given in Hirsch-Milnor [4] with a slight modification.

Since $\tau\left(P^{n}\right)$ and $f^{*}\left(\tau\left(M^{n}\right)\right)$ are stably equivalent, $\tau\left(P^{n}\right) \mid P^{-1}$ is equivalent to $f^{*}\left(\tau\left(M^{n}\right)\right) \mid P^{n-1}$. Let $c \in H^{n}\left(P^{n}, \pi_{n-1}(S O(n))\right.$ ) be the obstruction for extending the isomorphism $k:{ }_{\tau}\left(P^{n}\right)\left|P^{n-1} \approx f^{*}\left(\tau\left(M^{n}\right)\right)\right| P^{n-1}$ over $P^{n}$. (The coefficients are twisted).

The case $n=2$ is trivial, so we assume $n \geqq 4$.

Let $i: S O(n) \rightarrow S O(n+1)$ be the inclusion. Then

$$
0 \longrightarrow \operatorname{Ker} i_{*} \longrightarrow \pi_{n-1}(S O(n)) \stackrel{i_{*}}{\rightarrow} \pi_{n-1}(S O(n+1)) \rightarrow 0
$$

is exact, where $\operatorname{Ker} i_{*}=Z$ is generated by $\alpha=\alpha\left(\tau\left(S^{n}\right)\right)$ and each homomorphism is compatible with the operation of $\pi_{0}(0)=Z_{2}$. And the operation of the generator $\alpha_{0}$ of $\pi_{0}(0)$ is trivial on $\pi_{n-1}(S O(n+1))$ and $\alpha_{0}(\alpha)=-\alpha$ (Cf. Steenrod 
[10]). The above exact sequence induces the following exact sequence

$$
\begin{aligned}
& \rightarrow H^{n-1}\left(P^{n}, \pi_{n-1}(S O(n+1))\right) \rightarrow H^{n}\left(P^{n}, \text { Ker } i_{*}\right) \stackrel{j}{\longrightarrow} H^{n}\left(P^{n}, \pi_{n-1}(S O(n))\right) \\
& \left.\stackrel{i_{*}}{\longrightarrow} H^{n} ! P^{n}, \pi_{n-1}(S O(n+1))\right) \longrightarrow 0 .
\end{aligned}
$$

Since $H^{n-1}\left(P^{n}, \pi_{n-1}(S O(n+1))\right)=0$ or $Z_{2}$ and $H^{n}\left(P^{n}\right.$, Ker $\left.i_{k}\right)=Z, H^{n}\left(P^{n}\right.$, $\left.\operatorname{Ker} i_{k}\right)$ is mapped monomorphically into $H^{n}\left(P^{n}, \pi_{n-1}(S O(n))\right)$. If we choose an isomorphism $\left.k: \tau\left(P^{n}\right) \mid P^{n-1} \approx f^{*}\left(M^{n}\right)\right) \mid P^{n-1}$ carefully, $k$ can be extended over $P^{n}$ as an stable equivalence $\tau\left(P^{n}\right) \oplus 1 \approx f^{*}\left(\tau\left(M^{n}\right)\right) \oplus 1$, where 1 is a trivial line bundle over $P^{n}$. Thus $i_{k *}(c)=0$, and $c \in$ Image of $j$.

Considering the similar exact sequence over $S^{n}$, it is easy to see that $p: S^{n} \rightarrow P^{n}$ induces a homomorphism $p^{*}: H^{n}\left(P^{n}, \pi_{n-1}(S O(n))\right) \rightarrow H^{n}\left(S^{n}\right.$, $\left.\pi_{n-1}(S O(n))\right)$ which is a monomorphism on the image of $j$. Thus $c=0$ if and only if $p^{*}(c)=0$.

Now $p^{*}(c)$ is the primary obstruction for the existence of an isomorphism $p^{*}\left(\tau\left(P^{n}\right)\right) \approx p^{*} f^{*}\left(\tau\left(M^{n}\right)\right)$. But they are isomorphic as mentioned above. Thus $p^{*}(c)=0$, and it follows $c=0$. Therefore, $k$ can be altered so that it can be extended to an isomorphism $\tau\left(P^{n}\right) \approx f^{*}\left(\tau\left(M^{n}\right)\right)$ over whole $P^{n}$.

Corollary. Let $M^{n}$ be a homotopy real projective $n$-space. Then $M^{n} \times R^{k}$ is diffeomorphic to $P^{n} \times R^{k}$ for $k \geqq n+2$.

For this corollary we need only the stable equivalence of $\tau\left(P^{n}\right)$ and $f^{*}\left(\tau\left(M^{n}\right)\right)$ for a homotopy equivalence $f: P^{n} \rightarrow M^{n}$. This is shown at the beginning of the proof.

\section{References}

[1] J. F. Adams: On the non-existence of elements of Hopf invariant one, Ann. of Math. 72 (1960), 20-104.

[2] J. F. Adams: On the groups J(X)-II, Topology 3 (1965), 137-172.

[3] M. F. Atiyah: Thom complexes, Proc. London Math. Soc. (3) 11 (1961), 291-310.

[4] M. W. Hirsch and J. W. Milnor: Some curious involutions of spheres, Bull. Amer. Math. Soc. 70 (1964), 372-377.

[5] I. James and E. Thomas: An approach to the enumeration problem for non-stable vector bundles, Jour. of Math. and Mech. 14 (1965), 485-506.

[6] M. A. Kervaire and J. W. Milnor: Groups of homotopy spheres Part I, Ann. of Math. 77 (1963), 504-537.

[7] M. A. Kervaire: Some nonstable homotopy groups of Lie groups, I11. Jour. of Math. 4 (1960), 161-169. 
[8] B. Mazur: Stable equivalence of differentiable manifolds, Bull. Amer. Math. Soc. 68 (1961), 377-384.

[9] K. Shiraiwa: A remark on differentiable structures on real projective $(2 n-1)$-spaces, Nagoya Math. Jour. 27 (1966), 357-360.

[10] N. E. Steenrod: The topology of fibre bundles, Princeton, 1951.

[11] M. Takeuchi: A remark on the theorem of Peterson-Adachi, Jour. of Fac. of Sci., Univ, of Tokyo, 10 (1963-64), 124-128.

\section{Nagoya University}

